\title{
CIRUgÍA BARIÁTRICA EN EL HOSPITAL DE SAN JOSÉ, BOGOTÁ D.C. EXPERIENCIA Y RESULTADOS
}

\author{
Juan Camilo Ramírez MD*, Javier Darío Garzón MD**
}

\section{Resumen}

En los últimos años se ha dado gran importancia al tratamiento de la obesidad por el incremento de la incidencia a nivel mundial y a la falla del tratamiento médico en algunos tipos. Por eso hoy se practican con mayor frecuencia procedimientos de tipo bariátrico. Objetivo: describir la experiencia y resultados en el Hospital de San José de Bogotá D.C. entre enero 2006 y abril 2009. Métodos: cohorte de 146 pacientes con indicación de cirugía bariátrica en el período mencionado. Resultados: 54 hombres (37\%) con edad promedio de 54 años (DE: 10,5) y 92 mujeres (37\%) con 43 años (DE: 11). El promedio de índice de masa corporal de cirugía fue $43 \mathrm{k} / \mathrm{m}^{2}$ (DE: 5.1 ); veinte (13,7\%) se catalogaron superobesos. Las comorbilidades fueron hipertensión arterial $(\mathbf{4 3 , 1 \%})$, hipercolesterolemia $(\mathbf{4 0 , 4 \% )}$ y diabetes mellitus $(\mathbf{3 4 , 9 \% )}$. Hubo antecedente de cirugía abdominal en $\mathbf{3 4 , 2 \%}$. Todos los procedimientos corresponden a by pass gástrico, excepto uno de manga gástrica. El promedio de pérdida de peso entre el octavo y décimo mes fue $55 \%$ (DE: 9:9.1), con reducción de glicemia en ayunas y del perfil lipídico. No se obtuvieron datos en la totalidad de la población. La complicación más frecuente fue la ISO presente en doce $(8,2 \%)$, de los cuales ocho

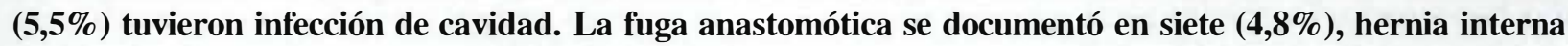
en tres $(2,1 \%)$, estenosis anastomótica y peritonitis secundaria en dos $(1,4 \%)$. No se reportaron hemoperitoneo, úlcera marginal ni hernia incisional. El tiempo quirúrgico promedio fue 106 minutos (DE: 22) y en 25 (17,1\%) se prolongó (mayor o igual a 120 minutos). El tiempo mediano de estancia hospitalaria fue cuatro días. La mortalidad global intrahospitalaria fue de siete $(4,7 \%)$, distribuida así: ningún caso en 2006 ( 35 cirugías), cuatro en 2007 (59 cirugías, 6,7\%), uno en 2008 ( 29 cirugías, $3,4 \%$ ) y dos en 2009 ( 22 cirugías, 9\%). Conclusiones: los pacientes llevados a cirugía bariátrica en el Hospital de San José presentaron una reducción adecuada de su exceso de peso en los primeros diez meses de posoperatorio, con clara tendencia a la reducción de glicemia, colesterol y triglicéridos.

Palabras clave: cirugía bariátrica, by pass gástrico, obesidad mórbida, porcentaje de pérdida de exceso de peso, índice de masa corporal.

Abreviaturas: IMC, índice de masa corporal; ISO, infección del sitio operatorio.

\section{BARIATRIC SURGERY AT HOSPITAL DE SAN JOSÉ BOGOTÁ DC. EXPERTISE AND RESULTS}

\section{Abstract}

Treatment for obesity has gained importance over the last few years as a result of increased global incidence and lack of medical therapeutic options for some types of overweight. Thus bariatric procedures are more frequently practiced nowadays. Objective: to describe expertise and results at Hospital de San José, Bogotá DC between January 2006 and April 2009. Methods: cohort of 146 patients with an indication for bariatric surgery during named period. Results: 54

Fecha recibido: junio I de 2010 Fecha aceptado: julio 14 de 2010

* Especialista en Cirugía General, Hospital de San José. Profesor Titular, Fundación Universitaria de Ciencias de la Salud. Bogotá DC. Colombia.
** Residente IV de Cirugía General, Fundación Universitaria de Ciencias de la Salud. Bogotá DC. Colombia. 
males (37\%), mean age 54 years (SD: 10.5) and 92 females (37\%), mean age 43 years (SD: 11). The mean body mass index of candidates to bariatric surgery was $43 \mathrm{k} / \mathrm{m}^{2}$ (SD: 5.1 ); 20 (13.7\%) were classified as extremely obese. Comorbid conditions included arterial hypertension (43.1\%), hyperlipidemia (40.4\%) and diabetes mellitus (34.9\%). There was an abdominal surgery history in $\mathbf{3 4 . 2 \%}$. All the procedures corresponded to a gastric bypass surgery except for one sleeve gastrectomy. Mean weight loss between an 8-month and 10.month post-surgical follow-up period was 55\% (SD: 9:9.1) with reduction of serum fasting glucose and lipid profile. Data from the total study population were not obtained. The most common complication was surgical site infection in 12 patients $(8.2 \%)$, of which 8 (5.5\%) had infection of the abdominal cavity. Anastomotic complications such as intestinal leaks was documented in 7 cases (4.8 $\%$ ), internal hernia in $3(2.1 \%)$, strictures with peritonitis in $2(1.4 \%)$. No cases of hemoperitoneum, marginal ulcers or incisional hernias were reported. Mean operating time was 106 minutes (SD: 22) and was greater in 25 (17.1\%) cases (= 120 minutes). Mean hospital stay was four days. The overall mortality rate in hospitalized patients was 7 (4.7\%), as follows: zero in 2006 (35 surgeries), 4 in 2007 (59 surgeries, $6.7 \%$ ), 1 in 2008 (29 surgeries, 3.4\%) and 2 in 2009 (22 surgeries, 9\%). Conclusions: Those who underwent bariatric surgery at Hospital de San José, presented adequate reduction of their overweight during the first ten-month postoperative follow-up period and frank reduction of glycemia, cholesterol and triglycerides.

Key words: bariatric surgery, gastric bypass surgery, morbid obesity, weight loss percent, body mass index.

\section{Introducción}

La dieta moderna rica en calorías, el sedentarismo y múltiples factores genéticos que aún se encuentran en estudio, han creado el ambiente ideal para el rápido aumento de la obesidad mórbida en nuestro medio. ${ }^{1,2} \mathrm{Sa}-$ bemos que en países como Estados Unidos uno de cada tres individuos de la población general es obeso. En otros lugares, como el norte de Europa, la prevalencia está cercana al 20\%. ${ }^{3}$ Nuestro país no es la excepción al problema creciente. La encuesta de salud y nutrición realizada en 2005 (ENSIN) sobre una muestra de 65.178 habitantes reportó cifras de obesidad del $13,7 \%$ en la población entre 18 y 64 años.

En ciertos tipos de obesidad el tratamiento médico exclusivo no genera cambios en el curso a largo plazo de esta enfermedad. ${ }^{4,5,6} \mathrm{Se}$ ha tratado en forma médica exclusiva (cambios en el estilo de vida y terapia farmacológica) la obesidad grado I (IMC $30-34.9 \mathrm{k} / \mathrm{m}^{2}$ ) $\sin$ encontrar una respuesta adecuada a largo plazo. El manejo quirúrgico se ha propuesto para el grado II (35-39.9 $\mathrm{k} / \mathrm{m}^{2}$ ) en presencia de comorbilidades derivadas de la obesidad. Así mismo, en la variedad mórbida (IMC $=40$ $\mathrm{k} / \mathrm{m}^{2}$ ) el beneficio de un procedimiento quirúrgico para reducir peso está establecido con claridad. ${ }^{7.8}$

Dado el incremento de la población obesa y el fracaso del manejo médico, cada día es mayor el número de procedimientos quirúrgicos de tipo bariátricoen nuestro medio y a nivel mundial. En Estados Unidos, por ejemplo, se reportaron cerca de 16.000 intervenciones en 1994, mientras que en 2005 se realizaron más de 170.000 y sigue en aumento. ${ }^{9}$ Se han clasificado estos procedimientos en tres grandes grupos: restrictivos, malabsortivos y mixtos. ${ }^{6,10}$ A pesar de que todos ellos tienen indicaciones, ventajas y diferentes resultados en cuanto a pérdida de peso a largo plazo, el de mejores resultados e impacto sobre las comorbilidades asociadas con la obesidad es el by pass gástrico por laparoscopia. " Por lo demás la cirugía bariátrica ha brindado mejoría en calidad de vida y sobrevida de los pacientes obesos.

Se ha demostrado que aquellos con obesidad mórbida sometidos a by pass gástrico por laparoscopia, reducen en un $5 \%$ el riesgo de muerte prematura, hay control metabólico de la diabetes mellitus tipo 2 en más de $90 \%$, se logra mejoría de cifras de tensión arterial y control lipídico, ${ }^{12} \sin$ contar los grandes beneficios en calidad de vida e imagen corporal que experimentan estos pacientes, lo cual ha hecho que en la actualidad sea el procedimiento bariátrico que se realiza con mayor frecuencia.

En este estudio presentamos la experiencia de cirugía bariátrica en el Hospital de San José de Bogotá D.C. Colombia. El objetivo primario es describir los resultados en los controles de peso en los primeros diez meses de seguimiento y del control metabólico. También se reportan las complicaciones quirúrgicas en el período intrahospitalario. 


\section{Materiales y métodos}

Se describen los resultados de una cohorte de pacientes con indicación de cirugía bariátrica, intervenidos entre enero de 2006 y abril de 2009. Se incluyeron todos los pacientes mayores de 18 años con indicación quirúrgica (independiente del procedimiento realizado) y se excluyeron los casos con antecedente de cirugía bariátrica previa. Los registros del servicio de cirugía y los hospitalarios se utilizaron para identificarlas intervenciones realizadas. Con la información de las historias clínicas (valoraciones de cirugía y clínica de obesidad) y de los reportes de juntas quirúrgicas, se describen las variables demográficas y los antecedentes de hipertensión arterial, diabetes mellitus 2, dislipidemia, síndrome apnea/hipopnea obstructiva del sueño y cirugía abdominal previa.

Se informan las complicaciones observadas en el período intrahospitalario registradas en la historia clínica, verificadas con los estudios paraclínicos y de imágenes diagnósticas cuando estaban disponibles. La presencia de fuga anastomótica descrita en la historia fue confirmada con el reporte del esofagograma o en la descripción del procedimiento de la reintervención. La presencia de material purulento en forma generalizada descrito en lasnotas de la reintervención definióel criterio de peritonitis secundaria. El registro de hallazgos laparoscópicos positivos ante la sospecha clínica de obstrucción intestinal, confirmó la presencia de hernias internas. Los pacientes con estenosis anastomótica fueron aquellos con diámetro menor de $1.2 \mathrm{~cm}$ en la anastomosis gastroyeyunal, ${ }^{12-14}$ documentado en la endoscopia de vía digestiva alta. La infección de sitio operatorio (ISO) fue valorada según criterios de SENIC. La mortalidad global se describe como muerte por cualquier causa durante la hospitalización. Reportamos el tipo de técnica quirúrgica, el tiempo quirúrgico y los días de estancia hospitalaria.

Por protocolo del servicio todos los pacientes llevados a cirugía bariátrica se citan a control a los ocho días, cuatro semanas y tres, seis, nueve y doce meses. Para fines del estudio se reportan datos sobre variables relacionadas con el cambio en el peso corporal y control metabólico (glicemia en ayunas, triglicéridos, LDL, HDL y cifras de presión arterial) obtenidas entre el segundo y cuarto mes posoperatorio y entre el octavo y décimo mes, teniendo en cuenta que algunos pacientes por su lugar de proce- dencia o trámites administrativos no pueden cumplir las citas establecidas. Las variables relacionadas con el peso corporal se describen en el período prequirúrgico y en dos controles posteriores. El IMC (peso/talla ${ }^{2}$ ) utilizado para la caracterización de la población fue 35 a $40 \mathrm{k} / \mathrm{m}^{2}$, obesidad mórbida 40.1 a $49.9 \mathrm{k} / \mathrm{m}^{2}$ y la categoría de superobesos cuando fue mayor o igual $50 \mathrm{k} / \mathrm{m}^{2} .{ }^{15} \mathrm{El} \mathrm{ex}$ ceso de peso se reporta como peso inicial-peso ideal. Para calcular el porcentaje de pérdida de exceso de peso se utilizó la fórmula pérdida de pesolexceso de peso por 100. ${ }^{6,16}$

En los registros se evaluó la presencia de úlcera marginal soportada en la endoscopia de vías digestivas altas en las consultas de control. Se verificó en las historias clínicas si existía reporte de hernia incisional en cualquier momento durante el seguimiento. Todos los análisis estadísticos se realizaron con STATA 10. Las variables categóricas se reportan como frecuencias absolutas y relativas. Las variables continuas se resumen como promedios y desviación estándar. Se utilizó la prueba de chi cuadrado o el test exacto de Fischer para evaluar la asociación de las variables clínicas y la mortalidad (hipótesis a dos colas $\alpha 0.05$ ).

\section{Resultados}

A partir de registros manuales del servicio de cirugía y datos estadísticos con códigos de egreso de gastroyeyunostomías, se identificaron 146 pacientes que fueron llevados a cirugía bariátrica; se confrontaron éstos con la base de datos de la clínica de obesidad y las juntas quirúrgicas. La Tabla 1 describe las características basales de la población de estudio. Cincuenta y cuatro pacientes (37\%) fueron hombres con edad promedio de 43 años (DE: 10.5) y 92 mujeres (63\%) con 43 años (DE: 11). El promedio de índice de masa corporal para toda la población previo al procedimiento quirúrgico fue de $43 \mathrm{k} / \mathrm{m}^{2}$ (DE: 5.1) y veinte $(13,7 \%$ ) que fueron catalogados como superobesos. La comorbilidad más frecuente fue la hipertensión arterial $(43,1 \%$, ) seguida de hipercolesterolemia $(40,4 \%)$ y diabetes mellitus $(34,9 \%)$. Se documentó antecedente de cirugía abdominal previa en $34,2 \%$ de los casos. Todos los procedimientos corresponden a by pass gástrico excepto un caso de manga gástrica. 
Tabla I. Características basales de pacientes de cirugía bariátrica

\begin{tabular}{|c|c|c|c|c|c|c|}
\hline \multirow[b]{2}{*}{$\begin{array}{l}\text { Edad, años promedio }(\mathrm{DE}) \\
\text { mínimo- máximo }\end{array}$} & \multicolumn{2}{|c|}{ Mujeres } & \multicolumn{2}{|c|}{ Hombres } & \multicolumn{2}{|c|}{ Total $n=146$} \\
\hline & $\begin{array}{r}92 \\
43.9 \\
21-\end{array}$ & $\begin{array}{l}(63,0 \%) \\
(11.5) \\
65\end{array}$ & $\begin{array}{r}54 \\
54 \\
23-\end{array}$ & $\begin{array}{l}(37 \%) \\
(10.5) \\
65\end{array}$ & $\begin{array}{r}43.5 \\
21-\end{array}$ & $\begin{array}{l}(11.1) \\
65\end{array}$ \\
\hline $\begin{array}{l}\text { IMC† } \mathrm{k} / \mathbf{m}^{2} \text {, promedio (DE) } \\
\text { mínimo-máximo } \\
35 \text { a } 40 \\
40.1 \text { a } 49.9 \\
\text { mayor o igual } 50\end{array}$ & $\begin{array}{r}43.3 \\
35 \\
24 \\
54 \\
14\end{array}$ & $\begin{array}{l}(5.2) \\
57 \\
(26.1) \\
(58.7) \\
(15.2)\end{array}$ & $\begin{array}{r}42.6 \\
35 \\
17 \\
31 \\
6\end{array}$ & $\begin{array}{l}(4.9) \\
52 \\
(31.5) \\
(57.4) \\
(11.1)\end{array}$ & $\begin{array}{l}43 \\
35 \\
41 \\
85 \\
20\end{array}$ & $\begin{array}{l}(5.1) \\
57 \\
(28.1) \\
(58.2) \\
(13.7)\end{array}$ \\
\hline \multicolumn{7}{|l|}{ Comorbilidades, n (\%) } \\
\hline $\begin{array}{l}\text { diabetes mellitus tipo } 2 \ddagger \\
\text { hipertrigliceridemia } \S \\
\text { hipercolesterolemia | } \\
\text { hipertensión arterial } \uparrow \\
\text { SAHOS* }\end{array}$ & $\begin{array}{l}30 \\
18 \\
37 \\
36 \\
20\end{array}$ & $\begin{array}{l}(32.6) \\
(19.5) \\
(40.2) \\
(39.1) \\
(21.0)\end{array}$ & $\begin{array}{l}21 \\
17 \\
22 \\
27 \\
19\end{array}$ & $\begin{array}{l}(38.8) \\
(31.4) \\
(40.7) \\
(50.0) \\
(35.1)\end{array}$ & $\begin{array}{l}51 \\
35 \\
59 \\
63 \\
39\end{array}$ & $\begin{array}{l}(34.9) \\
(23.9) \\
(40.4) \\
(43.1) \\
(26.7)\end{array}$ \\
\hline $\begin{array}{l}\text { Antecedente de cirugía abdominal previa } \\
\text { Laboratorios prequirúrgicos }\end{array}$ & 29 & $(31.5)$ & 21 & (38.9) & 50 & (34.2) \\
\hline $\begin{array}{l}\text { glicemia ayunas mg/dl, promedio (DE) } \\
\text { triglicéridos } \mathrm{mg} / \mathrm{dl} \text {, promedio (DE) } \\
\text { colesterol LDL, promedio (DE) } \\
\text { colesterol HDL, promedio (DE) }\end{array}$ & $\begin{array}{r}110 \\
186 \\
127 \\
48\end{array}$ & $\begin{array}{l}(26.8) \\
(71.7) \\
(22.2) \\
(12.5)\end{array}$ & $\begin{array}{r}112 \\
210 \\
127 \\
51\end{array}$ & $\begin{array}{l}(27.9) \\
(96.1) \\
(23.8) \\
(13.2)\end{array}$ & $\begin{array}{r}111 \\
195 \\
127 \\
49\end{array}$ & $\begin{array}{l}(27.4) \\
(82.1) \\
(22.7) \\
(12.7)\end{array}$ \\
\hline \multicolumn{7}{|l|}{ Cifras tensionales } \\
\hline $\begin{array}{l}\text { presión arterial sistólica, promedio (DE) } \\
\text { presión arterial diastólica, promedio (DE) }\end{array}$ & $\begin{array}{r}127.4 \\
81.7\end{array}$ & $\begin{array}{l}(13.4 \\
(7.7)\end{array}$ & $\begin{array}{r}131.9 \\
84.2\end{array}$ & $\begin{array}{l}(12.7) \\
(7.5)\end{array}$ & $\begin{array}{r}128 \\
82\end{array}$ & $\begin{array}{l}(13.3) \\
(7.7)\end{array}$ \\
\hline
\end{tabular}

SAHOS, síndrome de apnea obstructiva del sueño: con diagnóstico en historia clínica o polisomnograma que lo confirme; † IMC: índice de masa corporal, división del peso sobre la talla en metro ${ }^{2 ;} \ddagger D M$ tipo 2 : diagnóstico previo en historia clínica 0 condición presente antes del procedimiento quirúrgico; § hipertrigliceridemia: documentada por historia clínica; |hipercolesterolemia: documentada por historia clínica; \ hipertensión arterial: condición presente antes del procedimiento quirúrgico o del consumo de antihipertensivos.

Los cambios en el IMC, el porcentaje de pérdida de exceso de peso y resultados metabólicos documentados por laboratorio clínico se presentan distribuidos por sexo. (Tablas 2 y 3). En los hombres el promedio de IMC prequirúrgico fue $42.6 \mathrm{k} / \mathrm{m}^{2}$ (DE: 4.9 ), en su primer control el promedio fue $35.8 \mathrm{k} / \mathrm{m}^{2}$ (DE: 4.8 ) y en el control final $31.5 \mathrm{k} / \mathrm{m}^{2}$ (DE: 3.4 ). El promedio de porcentaje de pérdida de exceso de peso entre el octavo y décimo mes fue de 55\% (DE: 9.1). Se observó reducción en los niveles de glicemia en ayunas y perfil lipídico; sin embargo, no se obtuvieron datos para la totalidad de la población.
En las mujeres el promedio de IMC prequirúrgico fue de $43 \mathrm{k} / \mathrm{m}^{2}$ (DE: 5.2 ), observando reducción progresiva en los diferentes controles: $36 \mathrm{k} / \mathrm{m}^{2}$ (DE: 3.9) y $31.7 \mathrm{k} / \mathrm{m}^{2}$ (DE: 3.9 ) y $31.7 \mathrm{k} / \mathrm{m}^{2}$ (DE: 3.1 ) en el primer y en el último control. El comportamiento del porcentaje de pérdida de exceso de peso entre el octavo y décimo mes fue igual al descrito en los hombres 55\% (DE: 9.1) También se encontró una reducción progresiva de los niveles de glicemia, triglicéridos y LDL con limitación para el análisis por ausencia de registros. 
Tabla 2. Resultados de cirugía bariátrica en hombres

\begin{tabular}{|c|c|c|c|c|c|c|}
\hline $\mathrm{IMC} * \mathrm{k} / \mathrm{m}^{2}$, promedio $(\mathrm{DE} \dagger)$ & 42.6 & $(4.9)$ & 35.8 & $(4.8)$ & 31.5 & $(3.4)$ \\
\hline Exceso de peso $\ddagger k$, promedio (DE) & 57 & $(15.1)$ & 38 & $(12.5)$ & 25 & $(9.3)$ \\
\hline Pérdida de exceso de peso $\S, \%$ & & & 33 & $(10.9)$ & 55 & $(11.0)$ \\
\hline Glicemia ayunas $\Uparrow, \mathrm{mg} / \mathrm{dl}$, promedio (DE) & 112 & $(26.8)$ & 92 & $(15.5)$ & 89.9 & (9.9) \\
\hline Triglicéridos ** mg/dl, promedio (DE) & 210 & $(96.1)$ & 200 & (71.9) & 140.9 & 48.1 \\
\hline Colesterol LDL ††, promedio (DE) & 127 & 23.3 & 93.3 & $(11.8)$ & 128 & $(37.6)$ \\
\hline Colesterol HDLł‡, promedio (DE) & 51 & $(13.2)$ & 57 & $(14.1)$ & 49.6 & $(9.8)$ \\
\hline
\end{tabular}

* IMC: Índice de Quetelet peso k/talla $\mathrm{m}^{2}$. datos prequirúrgicos disponibles en 54 pacientes, datos control 2 a 4 meses, disponibles en $5 I$ pacientes, datos control 8 a 10 meses, disponibles en 51 pacientes; † desviación estándar; $¥$ exceso de peso en kilogramos previo al procedimiento quirúrgico, por encima de su peso ideal (peso inicial-peso ideal); § pérdida de peso expresado en porcentaje del exceso de peso calculada así: pérdida de peso/exceso de peso por 100; II glicemia: datos prequirúrgicos disponibles en 54 pacientes, datos control 2 a 4 meses, disponibles en 30 pacientes, datos control 8 a 10 meses, disponible en 39 pacientes; ** triglicéridos, datos prequirúrgicos disponibles en 54 pacientes, datos control 2 a 4 meses, disponibles en 7 pacientes, datos control 8 a 10 meses, disponibles en 14 pacientes; †† datos prequirúrgicos disponibles en 54 pacientes, datos control 2 a 4 meses, disponibles en 12 pacientes, datos control 8 a 10 meses disponibles en 1 I pacientes; 㧊 datos prequirúrgicos disponibles en 30 pacientes, datos control 2 a 4 meses, disponibles para 10 pacientes, datos control 8 a 10 meses disponibles en 15 pacientes.

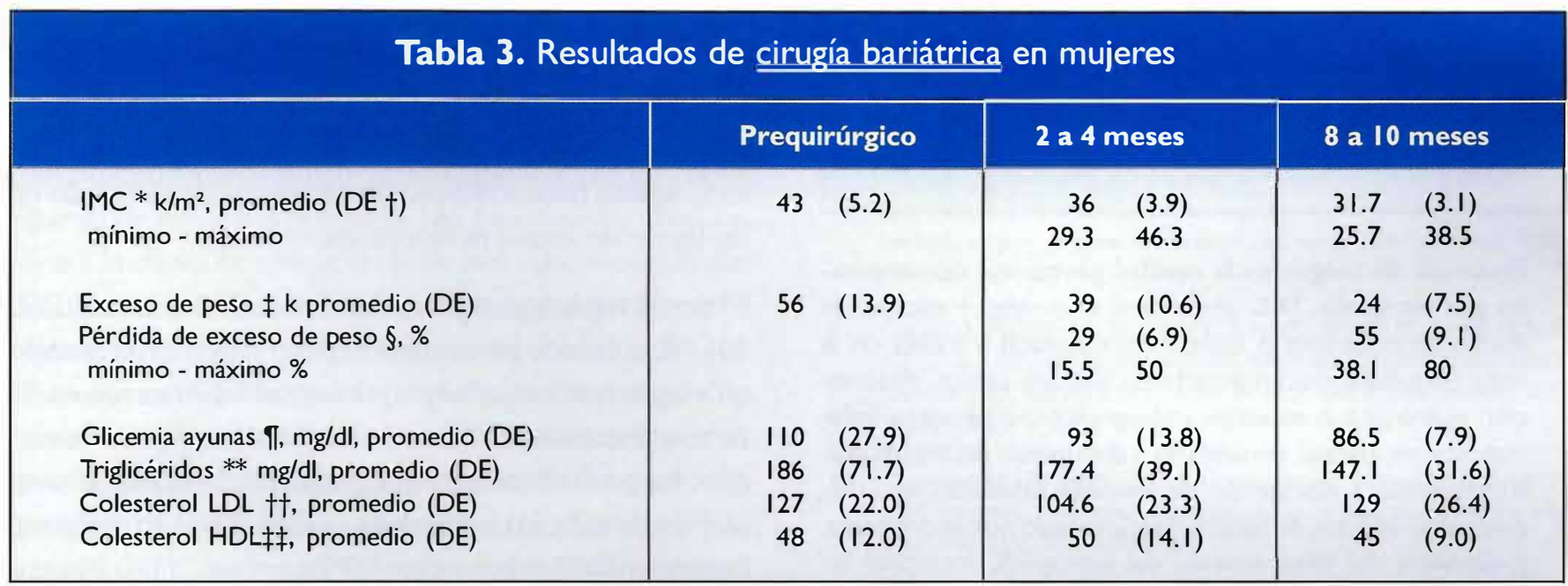

* IMC: Índice de Quetelet peso $\mathrm{k} /$ talla $\mathrm{m}^{2}$, datos prequirúrgicos disponibles en 92 pacientes, datos control 2 a 4 meses, disponibles en 88 pacientes, datos control 8 a 10 meses, disponibles en 88 ; $\dagger$ desviación estándar; $\ddagger$ exceso de peso en kilogramos antes del procedimiento quirúrgico, por encima de su peso ideal peso inicial-peso ideal; $\S$ pérdida de peso expresada en porcentaje del exceso de peso calculada así: pérdida de peso/exceso de peso por 100; \l glicemia; datos prequirúrgicos disponibles en 92 pacientes, datos control 2 a 4 meses, disponibles en 64; datos control 8 a 10 meses, disponibles en 60; ** triglicéridos; datos prequirúrgicos disponibles en 92 pacientes, datos control 2 a 4 meses, disponibles en 18; datos control 8 a 10 meses, disponibles en 17; †† LDL: datos prequirúrgicos disponibles en 92; datos control 2 a 4

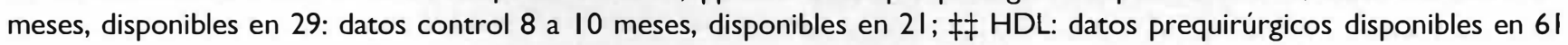
pacientes, datos control 2 a 4 meses, disponibles en 21 ; datos control 8 a 10 meses, disponibles en 24 . 
La complicación quirúrgica más frecuente fue la ISO presente en doce pacientes $(8,2 \%)$, de lós cuales ocho $(5,5 \%)$ tuvieron infección de cavidad. La fuga anastomótica se documentó en siete $(4,8 \%)$, hernia interna en tres $(2,1 \%)$, estenosis anastomótica y peritonitis secundaria en dos $(1,4 \%)$. No se reportaron casos de hemoperitoneo, úlcera marginal ni hemia incisional (Tabla 4). La fuga anastomótica se documentó en cuatro

\section{Tabla 4. Desenlaces $n=\mid 46$}

\begin{tabular}{|c|c|c|}
\hline Tiempo quirúrgico mn, promedio (DE*) & 106 & $(22.0)$ \\
\hline mínimo - máximo & 50 & -160 \\
\hline tiempo $=120$ minutos, $n(\%)$ & 25 & $(17.1)$ \\
\hline Complicaciones, $\mathrm{n}(\%)$ & 15 & $(10.2)$ \\
\hline hemoperitoneo * & - & - \\
\hline fuga anastomótica $\dagger$ & 7 & $(4.8)$ \\
\hline peritonitis secundariał & 2 & $(1.4)$ \\
\hline úlcera marginal | & - & - \\
\hline hernia interna $\|$ & 3 & $(2.1)$ \\
\hline estenosis anastomótica** & 2 & (1.4) \\
\hline 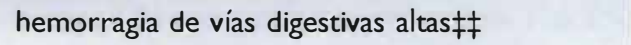 & 1 & $(0.7)$ \\
\hline Infección de sitio operatorio, n (\%) & 12 & (8.2) \\
\hline superficial & 4 & $(2.7)$ \\
\hline profunda & - & - \\
\hline cavidad & 8 & $(5.5)$ \\
\hline Estancia hospitalaria en dias, mediana(RIQ) & 4 & $(4-5)$ \\
\hline mínimo-máximo & 2 & -34 \\
\hline Mortalidad global n (\%) & 7 & $(4.7)$ \\
\hline
\end{tabular}

* Presencia de sangre en la cavidad peritoneal documentado por ecografía, TAC abdominal o cirugía; $†$ escape de medio de contraste o contenido intestinal a través de la línea de sutura, documentado en historia clínica, descripción quirúrgica o estudios radiográficos; $\ddagger$ proceso inflamatorio peritoneal, secundario a disrupción del tracto gastrointestinal; | disrupción de mucosa intestinal en cualquier sitio de línea de sutura diagnosticado por endoscopia; Il ausencia del paso normal del contenido intestinal en forma mecánica, diagnosticado por radiografía de abdomen, TAC de abdomen, cirugía o documentado en historia clínica; T diagnóstico de infección de sitio operatorio hecha por un cirujano en los primeros 30 días después del procedimiento quirúrgico, o aislamiento de germen de secreción de la herida quirúrgica; ${ }^{* *}$ disminución en el calibre de la anastomosis, menor de $1.2 \mathrm{~cm}$ documentada por endoscopia o vías digestivas altas, asociada con clínica compatible; 抽 documentación en historia clínica de diagnóstico confirmado por endoscopia. casos por laparoscopia y tres por esofagograma, dos fueron manejados en forma médica exclusiva con reposo intestinal, antibioticoterapia y nutrición parenteral por 14 días, con adecuada evolución sin reintervención quirúrgica.Dos requirieron reintervención, manejados mediante laparoscopia exclusiva con sistema de drenaje, refuerzo de la anastomosis y antibioticoterapia. Los otros pacientes presentaron como consecuencia de la fuga anastomótica peritonitis generalizada, requiriendo por su condición séptica laparotomía más drenaje de la peritonitis, lavados quirúrgicos a demanda y manejo en cuidado intensivo. Dos pacientes que presentaron peritonitis generalizada requirieron laparotomía más abdomen abierto y lavados quirúrgicos a demanda. Los ocho casos de infección de cavidad, fueron los mismos en quienes se documentaron fugas anastomótica y peritonitis generalizada, manejados como se explicó antes.

De los tres pacientes que presentaron hernias internas, dos se manejaron mediante laparoscopia exclusiva, logrando reducción satisfactoria sin complicaciones asociadas. En uno la fuga anastomótica se debió al aumento de la presión retrógradarequiriendo conversión del procedimiento a laparotomía y manejo de la peritonitis en la unidad de cuidado intensivo. La estenosis anastomótica se documentó de forma endoscópica, ambos casos fueron manejados en forma satisfactoria con dilataciones. Uno presentó hemorragia de vía digestiva alta durante la estancia en cuidado intensivo que se catalogó como gastritis aguda hemorrágica, sin presencia de sangrado en las líneas de sutura ni úlceras marginales.

El tiempo quirúrgico promedio fue de 106 minutos (DE: $22)$. Veinticinco pacientes $(17,1 \%)$ requirieron tiempo quirúrgico prolongado (mayor o igual a 120 minutos). El tiempo mediano de estancia hospitalaria fue de cuatro días. La mortalidad global intrahospitalaria fue de siete (4,7\%), distribuida así: ningún caso en 2006 (36 cirugías), cuatro en 2007 ( 59 cirugías, 6,7\%), uno en 2008 (29 cirugías, 3,4\%) y dos en 2009 (22 cirugías, 9\%). No se encontró asociación entre diabetes, superobesidad (IMC=50 $\mathrm{k} / \mathrm{m}^{2}$ ), antecedente de cirugía abdominal e hipertensión arterial con la mortalidad. Existe asociación estadística significativa entre ISO de cavidad (p 0.000), complicaciones quirúrgicas ( $\mathrm{p} 0.000)$ y tiempo quirúrgico prolongado ( $\mathrm{p}=0.007)$ con la mortalidad intrahospitalaria. El tiempo quirúrgico prolongado también se encontró aso- 
ciado con la presencia de ISO $(\mathrm{p}=0.017)$ pero no con las complicaciones quirúrgicas $(\mathrm{p}>0.05)$.

\section{Discusión}

Buchwald reportó que los pacientes sometidos a by pass gástrico por laparoscopia pierden hasta un $80 \%$ de su exceso de peso en el transcurso de los dos primeros años de posoperatorio. ${ }^{1,16}$ Nuestros resultados muestran adecuada reducción de exceso de peso al encontrar que en promedio perdieron el $55 \%$ antes de completar un año de cirugía. La complicación intrahospitalaria más frecuente después de la infección del sitio operatorio de cavidad fue la fuga anastomótica, hallazgo que concuerda con los reportes de la literatura, que revela complicaciones derivadas de las anastomosis hasta en un $5 \% .^{9}$ La peritonitis secundaria en las diferentes series es la que más se asocia con mortalidad. ${ }^{17} \mathrm{La}$ hernia interna ocupa el tercer lugar en frecuencia de aparición en el posoperatorio. Algunas series la consideran como complicación tardía, pero en nuestro estudio se manifestó en forma aguda. $^{18}$

La mortalidad en nuestra serie fue de $4,7 \%$, superior a la literatura, la cual en general es menor del $1 \%{ }^{19}$ pero sólo consideran la derivada del procedimiento quirúrgico. Nosotros reportamos mortalidad global, sin importar si la causa de muerte estuvo relacionada con complicaciones del procedimiento quirúrgico. Debe tenerse en cuenta que se incluyen los primeros 146 casos que corresponden a la curva de aprendizaje de tres cirujanos diferentes, la cual está determinada por los primeros 40 procedimientos ${ }^{20}$ que corresponden a más del $80 \%$. La mediana de estancia hospitalaria global fue de cuatro días, siendo similar a la mayoría de publicaciones; sin embargo, se evidenció que en algunos pacientes fue prolongada (datos no mostrados) atribuida a trámites logísticos de desplazamiento aéreo y otros procesos administrativos que no fueron objeto de estudio.

Algunos reportan que la presencia de diabetes mellitus, cirugía abdominal previa y superobesidad cursa con mayor riesgo de muerte en el posoperatorio de by pass gástrico. ${ }^{21}$ En nuestra serie llama la atención que ninguna de estas variables tuvo relación estadística significativa. Los que fallecieron tuvieron un tiempo quirúr- gico mayor de 120 minutos, se relacionaron con la presencia de infección del sitio operatorio de cavidad y en general con complicaciones propias del procedimiento quirúrgico.

La cirugía bariátrica aunque ha demostrado grandes beneficios en la resolución de comorbilidades y un adecuado control de peso a corto y largo plazo, es un procedimiento que tiene riesgos no despreciables con complicaciones que pueden tener alta mortalidad. En esta serie se reportan los beneficios en reducción de peso, lo cual tendrá un impacto clínico importante. Este es un informe inicial de nuestra experiencia local, con algunas dificultades y pérdidas de información, que requiere publicaciones futuras.

\section{Referencias}

1. Buchwald H, Avidor Y, Braunwald E, Jensen MD, Pories W, Fahrbach K, et al. Bariatric surgery: a systematic review and meta-analysis. JAMA. 2004 Oct $13 ; 292(14): 1724-37$.

2. Buchwald H, Estok R, Fahrbach K, Banel D, Jensen MD, Pories WJ, et al. Weight and type 2 diabetes after bariatric surgery: systematic review and meta-analysis. Am J Med. 2009 Mar; 122(3):248-56.

3. Bult MJ, van DT, Muller AF. Surgical treatment of obesity. Eur J Endocrinol. 2008 Feb; 158(2):135-45.

4. Campos GM, Ciovica R, Rogers SJ, Posselt AM, Vittinghoff E, Takata M, et al. Spectrum and risk factors of complications after gastric bypass. Arch Surg. 2007 Oct;142(10):969-75.

5. Christou N, Efthimiou E. Five-year outcomes of laparoscopic adjustable gastric banding and laparoscopic Roux-en-Y gastric bypass in a comprehensive bariatric surgery program in Canada. Can J Surg. 2009 Dec; 52(6): E249E258.

6. Garza CA, Pellikka PA, Somers VK, Sarr MG, Seward JB, Collazo-Clavell $\mathrm{ML}$, et al. Major weight loss prevents long-term left atrial enlargement in patients with morbid and extreme obesity. Eur J Echocardiogr. 2008 Sep;9(5):587-93.

7. Campos GM, Ciovica R, Rogers SJ, Posselt AM, Vittinghoff E, Takata M, et al. Spectrum and risk factors of complications after gastric bypass. Arch Surg. 2007 Oct;142(10):969-75.

8. Del Campo MF, de Frutos AJ, Sanchez FA. [Acute respiratory failure immediately following surgery for morbid obesity]. Arch Bronconeumol. 2008 Aug; 44(8):449-50.

9. Maggard MA, Shugarman LR, Suttorp M, Maglione M, Sugerman HJ, Livingston EH, etal. Meta-analysis: surgical treatment of obesity. Ann Intern Med. 2005 Apr 5;142(7):547-59.

10. Iannelli A, Dainese R, Piche T, Facchiano E, Gugenheim J. Laparoscopic sleeve gastrectomy for morbid obesity. World J Gastroenterol. 2008 Feb 14;14(6):821-7. 
11. van WB, Berends FJ, Van RB, Janssen IF. Revision of failed laparoscopic adjustable gastric banding to Roux-en-Y gastric bypass. Obes Surg. 2006 Feb;16(2):137-41.

12. Still CD, Benotti P, Wood GC, Gerhard GS, Petrick A, Reed M, et al. Outcomes of preoperative weight loss in high-risk patients undergoing gastric bypass surgery. Arch Surg. 2007 Oct;142(10):994-8.

13. Eubanks S, Edwards CA, Fearing NM, Ramaswamy A, de la Torre RA, Thaler KJ, et al. Use of endoscopic stents to treat anastomotic complications after bariatric surgery. J Am Coll Surg. 2008 May;206(5):935-8.

14. Frutos MD, Lujan J, Garcia A, Hernandez Q, Valero G, Gil J, et al. Gastrojejunal anastomotic stenosis in laparoscopic gastric bypass with a circular stapler $(21 \mathrm{~mm})$ : incidence, treatment and long-term follow-up. Obes Surg. 2009 Dec; $19(12): 1631-5$

15. Ikramuddin S, Klingman D, Swan T, Minshall ME. Cost-effectiveness of Roux-en-Y gastric bypass in type 2 diabetes patients. Am J Manag Care. 2009 Sep;15(9):607-15.

16. Lakdawala MA, Bhasker A, Mulchandani D, Goel S, Jain S. Comparison between the results of laparoscopic sleeve gastrectomy and laparoscopic roux- en- $Y$ gastric bypass in the indian population: a retrospective 1 year study. Obes Surg. 2010 Jan;20(1 ): 1-6.

17. Mathier MA, Zhang J, Ramanathan RC. Dramatic functional improvement following bariatric surgery in a patient with pulmonary arterial hypertension and morbid obesity. Chest. 2008 Mar; 133(3):789-92.

18. Pories WJ. Bariatric surgery: risks and rewards. J Clin Endocrinol Metab. 2008 Nov;93(11 Suppl 1):S89-S96.

19. McCarty TM, Arnold DT, Lamont JP, Fisher TL, Kuhn JA. Optimizing outcomes in bariatric surgery: outpatient laparoscopic gastric bypass. Ann Surg. 2005 Oct; 242(4):494-8.

20. Mognol P, Chosidow D, Marmuse JP. Laparoscopic conversion of laparoscopic gastric banding to Roux-en- $\mathrm{Y}$ gastric bypass: a review of 70 patients. Obes Surg. 2004 Nov; 14(10): 1349-53.

21. Omalu BI, Ives DG, Buhari AM, Lindner JL, Schauer PR, Wecht CH, et al. Death rates and causes of death after bariatric surgery for Pennsylvania residents, 1995 to 2004. Arch Surg. 2007 Oct; 142(10):923-8.

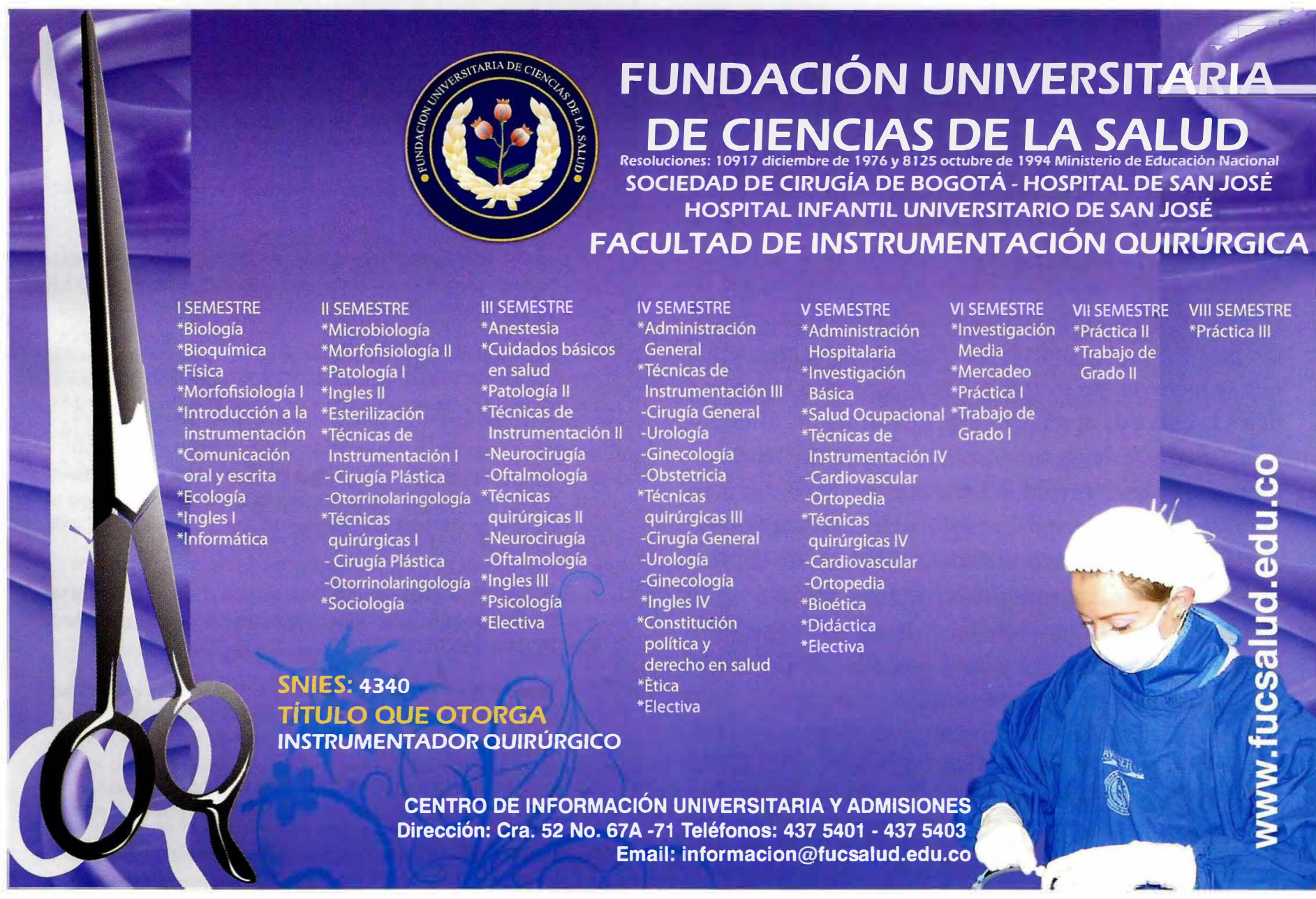

